\title{
Undiagnosed paraganglioma; A challenge during laparoscopic retroperitoneal resection
}

\author{
Alexander Heinze ${ }^{1,3}$, Periklis Nikomanis ${ }^{2}$, Ferdinand Petzold ${ }^{2}$, Jens Jochen Rassweiler ${ }^{1}$, \\ Ali Serdar Goezen ${ }^{1}$ \\ ${ }^{1}$ SLK Kliniken Heilbronn, Department of Urology, University of Heidelberg, Germany; \\ ${ }^{2}$ SLK Kliniken Heilbronn, Department of Anaesthesiology, University of Heidelberg Germany; \\ ${ }^{3}$ School of Medicine, Universidad Nacional Autónoma de México, México City.
}

\begin{abstract}
Summary Objective: Report our experience of the management of a patient with undiagnosed retroperitoneal paraganglioma and the intraoperative complications that the theatre team faced.

Case report: We present a case of a 36-year-old patient who during oncological follow-up for a previous diagnosis of parotid acinar cell carcinoma was incidentally identified as having an interaortocaval tumour. Following routine preoperative assessment the patient was arranged to undergo a laparoscopic retroperitoneal tumour resection. After minimal tumour manipulation the patient developed cardiac rhythm abnormalities and became hypertensive. The tumour was successfully removed laparoscopically after a cautious inter-aortocaval dissection. Abruptly, prior to extraction of the tumour containing endobag, the patient developed cardiac arrest. Following 35 minutes of life support measures there was a return of spontaneous circulation. The endobag was laparoscopically removed from the abdominal cavity 24 hours later using the initial operative port sites. The patient's progression was satisfactory and he could be discharged six days postoperatively.

Conclusions: Asymptomatic undiagnosed paragangliomas represent a real challenge during laparoscopic operations.

Haemodynamic changes and life-threatening events can arise acutely intraoperatively, where an immediate and coordinated response of the whole theatre team may be required to avoid fatal outcomes.
\end{abstract}

KEY WORDS: Paraganglioma; Laparoscopic approach; Intraoperative complications.

Aubmitted 25 March 2018; Accepted 7 May 2018

\section{INTRODUCTION}

Paragangliomas are defined as neuroendocrine tumors (NET) which may or may not produce catecholamines. These tumors emerged out of chromaffin cells from the neural crest. These type of tumors are distributed along the sympathetic and parasympathetic chains. The most common location is retroperitoneal accounting for up to $77 \%$ often pictured as a mass around the corpora paraaortica (1). Surgical resection represents the only available curative treatment for these tumors.

The laparoscopic approach has proved to be a safe and effective technique for retroperitoneal tumor resection in experienced hands $(2,3)$. But serious challenges and complications can arise during these operations both for urology and anesthesia teams when the correct diagnosis is not suspected preoperatively.

\section{Clinical case}

We present the case of a 36-year-old man who attended our urological service. The patient received a diagnosis of parotid acinar cell carcinoma nine months prior, and during oncological follow-up was incidentally identified through the use of computed tomography (CT) imaging which revealed a $4.8 \mathrm{~cm}$ lesion with heterogeneous softtissue density in an interaortocaval localization without any sign of vascular infiltration, hence his referral to our urology service. The patient was asymptomatic with a background history of hypothyroidism, gastrooesophageal reflux disease, parotidectomy and neck dissection. Physical examination and vital sign parameters were unremarkable and routine laboratory blood tests were within normal ranges. The patient was arranged to undergo laparoscopic retroperitoneal tumor resection following preoperative assessment. After positioning the patient in supine position, we favored a transperitoneal 4-trocars approach, and establishment of a pneumoperitoneum up to $14 \mathrm{~mm} / \mathrm{hg}$ of pressure. The access to the retroperitoneum was achieved by incision the white line of Toldt and by displacing medially the ascendant colon. The interaortocaval localized tumor was then visualized and a careful dissection of the surrounding big vessels and tissue was undertaken. The dissection was carried out with a minimal blood loss. After a minimal tumor manipulation the patient developed cardiac rhythm abnormalities - possibly atrioventricular block - and rapidly became hypertensive with a systolic blood pressure of $250 \mathrm{mmHg}$ and a tachycardia of $160 \mathrm{bpm}$.

The patient was initially administered $\beta$-blockers and an attempt to deepen the anesthesia using opiates was undertaken, unfortunately with poor response.

The patient subsequently developed respiratory failure with hypoxia and clinically was found to have pulmonary edema. Meanwhile surgery was accomplished successfully and the resected specimen was placed into an endobag. Abruptly prior to extraction of the endobag through the paraumblical optic trocar incision the patient developed cardiac arrest, indicating pulseless

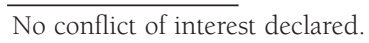


electrical activity on electrocardiography. Therefore, resuscitation maneuvers' were employed immediately following standardized life support protocols. The port incisions were closed rapidly while operating team begun manual chest compressions - initially by hand and subsequently aided with the mechanical chest compression device "LUCAS'TM (physio Control Inc. Redmond WA)". Following 35 minutes of resuscitation measures there was a return of spontaneous circulation (ROSC) with sinus rhythm, and the patient was transferred to the Operative Intensive Care Unit (OPI) where respiratory and cardiovascular support was offered through ventilation and catecholamine perfusions. Cardiac rhythm and function was assessed with electrocardiography and echocardiography, which initially showed findings consistent with post-resuscitation reduced left ventricular function. Follow-up echocardiography indicated a possible early stage hypertensive cardiomyopathy. Coronary angiography excluded any coronary artery disease. Chest CT of the patient's identified mild pulmonary edema and postresuscitation atelectasis. A control CT of the patient's abdomen showed the endobag containing the resected tumor and no other acute pathology. Further laparoscopic intervention using the same port places was carried out 24 hours after the initial surgery in order to remove of the endobag with resected tumor.

The endobag has been visualized easily in the abdominal cavity and could be removed after minimal manipulation safely through the paraumblical optic trocar incision, Tumor pathology and histology results reported a $4.5 \mathrm{~cm}$ x $4.5 \mathrm{~cm} \times 2 \mathrm{~cm}$ tissue of yellow brownish color surrounded by a thin capsule. Inmunohistology was performed being positive for chromogranin A and synaptophysin and negative for pancytoqueratine, protein S-100 and melanina A. This findings were compatible with characteristics of paraganglioma tumors.
The patient remained on the OPI for a further 72 hours. During this period, the patient showed clinical stabilization and improvement with no lasting neurological or end-organ damage evident. Therefore, the patient was transferred to the urological ward from which he was formally discharged six days post-operatively. Further followup will be carried out by urology department and further genetic analysis will be performed by genetic department.

\section{Conclusions}

Asymptomatic undiagnosed paragangliomas represents a real perioperative challenge. Ideally, patients with suspected diagnoses of paraganglioma or pheochromocytoma should receive adequate preoperative management for optimal surgical conditions. Laparoscopic approach should be considered in experienced hands due to its well known advantages. Nevertheless risk of acute hemodynamic changes and life-threatening events can arise in these subgroups of patients; for this reason the entire operating team should be prepared in order to avoid fatal outcomes.

\section{References}

1. Purnell S, Sidana A, Maruf M, et al. Genitourinary paraganglioma: Demographic, pathologic, and clinical characteristics in the surveillance, epidemiology, and end results database (2000-2012). Urol Oncol. 2017; 35:457.e9-457.e14.

2. Abe T, Sazawa A, Harabayashi T, et al. Laparoscopic resection of paraaortic/paracaval neurogenic tumors: surgical outcomes and technical tips. Surg Endosc. 2016; 30:4640-4645.

3. Ping W, Hong Zhou M, Jie Q, et al. Laparoscopic Resection of Retroperitoneal Paragangliomas: A Comparison with Conventional Open Surgical Procedures. J Endourol. 2016; 30:69-74.

\section{Correspondence}

Alexander Heinze, MD

Periklis Nikomanis, MD

Ferdinand Petzold, MD

Jens Jochen Rassweiler, MD

Ali Serdar Goezen, MD FEBU (Corresponding Author) ali.goezen@slk-kliniken.de SLK-Kliniken Heilbronn, Department of Urology Am Gesundbrunnen 20-26, D-74078 Heilbronn, Germany 